\title{
A Large Glomus Tumor of the Face: A Case Report
}

Javad Yazdani ${ }^{1}$, Mohammad Ali Ghavimi ${ }^{1}$, Reza Khorshidi ${ }^{1}$, Kourosh Taheri Talesh ${ }^{1}$, Ali Mortazavi ${ }^{2}$, Amin Nourizadeh $^{3^{*}}$

1. Department of Oral and Maxillofacial Surgery, Faculty of Dentistry, Tabriz University of Medical Sciences, Tabriz, Iran

2. Department of Oral and Maxillofacial Surgery, Faculty of Dentistry, Ardabil University of Medical Sciences, Ardabil, Iran

3. Department of Prosthodontics, Faculty of Dentistry, Tabriz University of Medical Sciences, Tabriz, Iran

\begin{abstract}
Glomus tumors are neoplasms arising from the glomus bodies, neuromyoarterial units found within the reticular dermis functioning as a specialized arteriovenous anastomosis. These rare tumors are benign and located anywhere in the body, and their cells closely resemble those of a normal glomus body. In this article, we report occurrence and administered treatment for a large glomangioma in the malar region of an 8-year-old male.
\end{abstract}

Keywords: Glomus tumor, glomus body, face

\section{Introduction}

The glomus body is an apparatus between the arterial and venous system located in the skin (1). Glomus tumors are benign tumors whose cells closely resemble those of a normal glomus body (2). They belong to the group of perivascular tumors, first described by Masson in $1924(1,3)$. Glomus tumors are uncommon and account for $1-5 \%$ of all soft-tissue tumors of the hand (4). The location of a glomus tumor can vary widely. Similar to the cutaneous neoplasms, glomus tumors usually occur in the subungual regions of digits or in the deep dermis of the palm, wrist, and forearm (5). However, their occurrence in extracutaneous sites such as bone, nerve, mediastinum, stomach, colon, trachea, and lungs has also been reported in previously published literature (5). Particularly, this tumor is very rare in the face. To date, few cases of glomus tumor in the cheek eyelid have been documented in the literature and therefore very little is known about the biological behavior and the demographic trend of this unusual lesion. Here, we report occurrence and subsequent treatment of a large glomus tumor in the malar region of an 8-year-old male.

\section{Corresponding author:}

Amin Nourizadeh

Department of Prosthodontics, Faculty of Dentistry, Tabriz University of Medical Sciences, Tabriz, Iran E-mail: nourizadeh.amin@gmail.com Tel: +989144129518

Receive date: 2015-06-29| Accept date: 2015-07-25| Publish date: 2015-08-04

DOI: 10.7575/aiac.abcmed.15.03.04.10

\section{Al}




\section{Case Report}

An 8-year-old ASA I male patient with a chief complaint of right malar tumor was admitted to the Department of Maxillofacial Surgery at Tabriz University of Medical Sciences. The patient had no history of systemic problems but he noted a facial trauma one year before his reference. The patient's clinical findings included severe swelling of right malar, buccal and infraorbital spaces without any visual or auditory disturbance (Figure 1). The overlying skin was normal and was extremely painful on palpation. There was no facial paresthesia and trismus. Intraoral examination revealed an expansile exophytic lesion at the right buccal vestibule. Oral lesions were exposed to dental trauma and involved mid-palatal suture. Radiographic evaluation with spiral computed tomography (CT) scan revealed a big mass in the buccal and infra-temporal spaces (Figure 2). The mass occupied the whole maxillary sinus space leading to resorption of tuberosity and lateral nasal wall. The mass superiorly spread to the orbital floor and posteriorly to the pterygomaxillary fissure. Laboratory tests including complete blood count, biochemistry, calcium, phosphorus, and alkaline phosphatase were within normal range. Under general anesthesia, the tumor was resected by an intraoral approach due to cosmetic reasons and to protect the facial nerve branches. In the posterior region, the tumor did not have any distinct border, and in the buccal space it was attached to the adjacent muscles. Parts of the sinus wall and maxillary tuberosity and hard palate were resorbed due to the tumor expansion. No abnormal bleeding was noted during surgery. In the pathologic evaluation, fibroconnective tissue and skeletal muscle with diffuse infiltration of round cells with mildly pleomorphic nuclei were seen. Scanty to moderate cytoplasm, high nucleus-tocytoplasm ratio, scattered mitosis and vascular background were also illustrated (Figure 3 ). The neoplastic cells express immunoreactivity as below: positive for actin, vimentin and CD34 in some cells, and negative for CD45, S100, and desmin. In the postoperative follow-up, the patient had some degree of trismus due to adherence of tumor to the masticatory muscles.

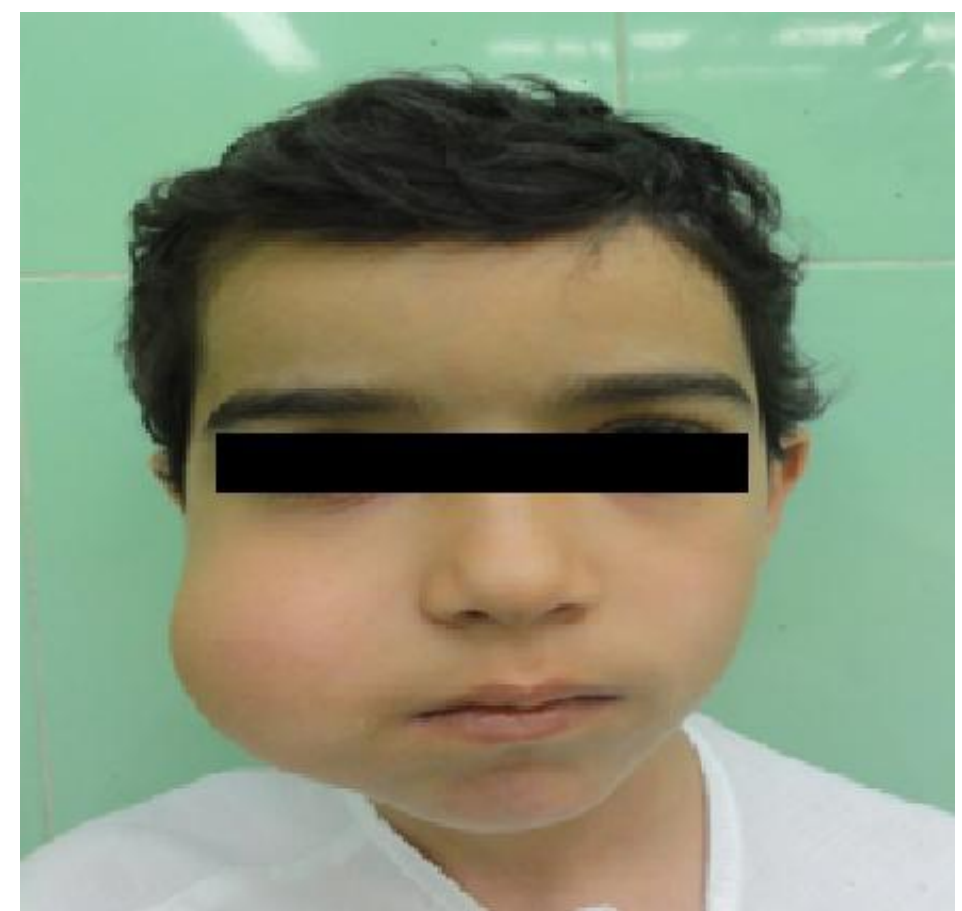

Figure 1: Severe swelling of right malar, buccal and infraorbital spaces in an 8-year old male 


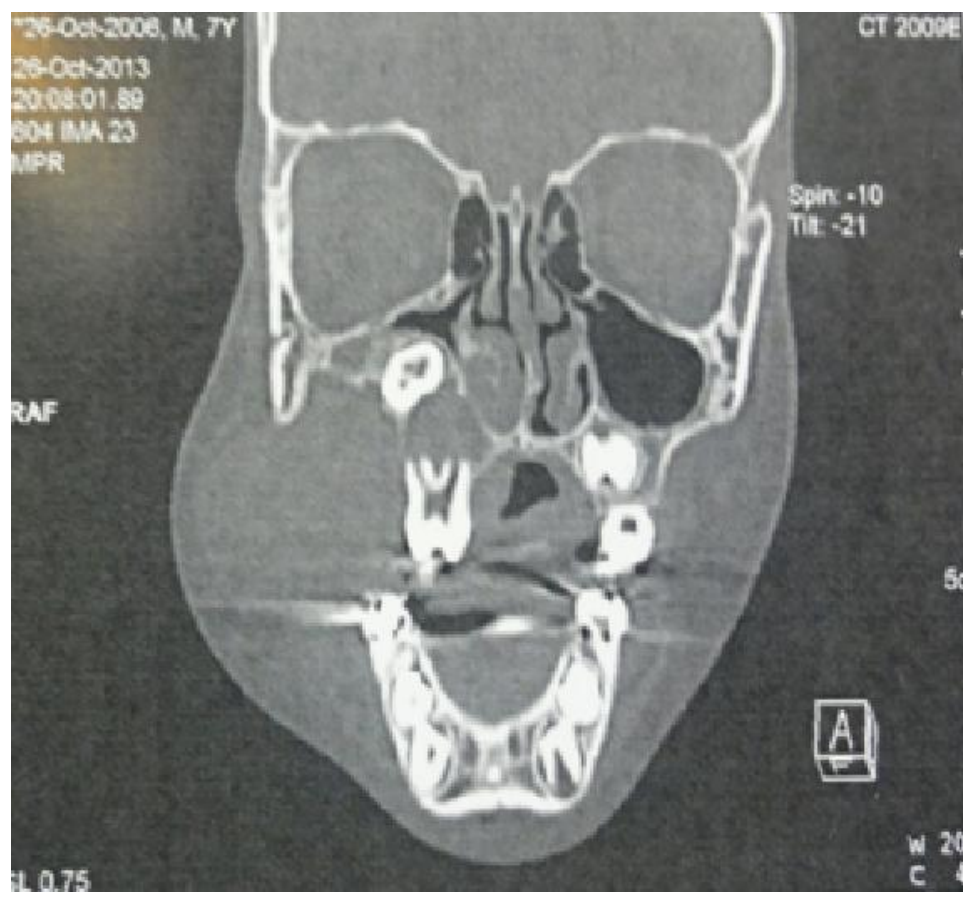

Figure 2: Spiral computed tomography scan showing a big mass in the buccal and infra-temporal spaces

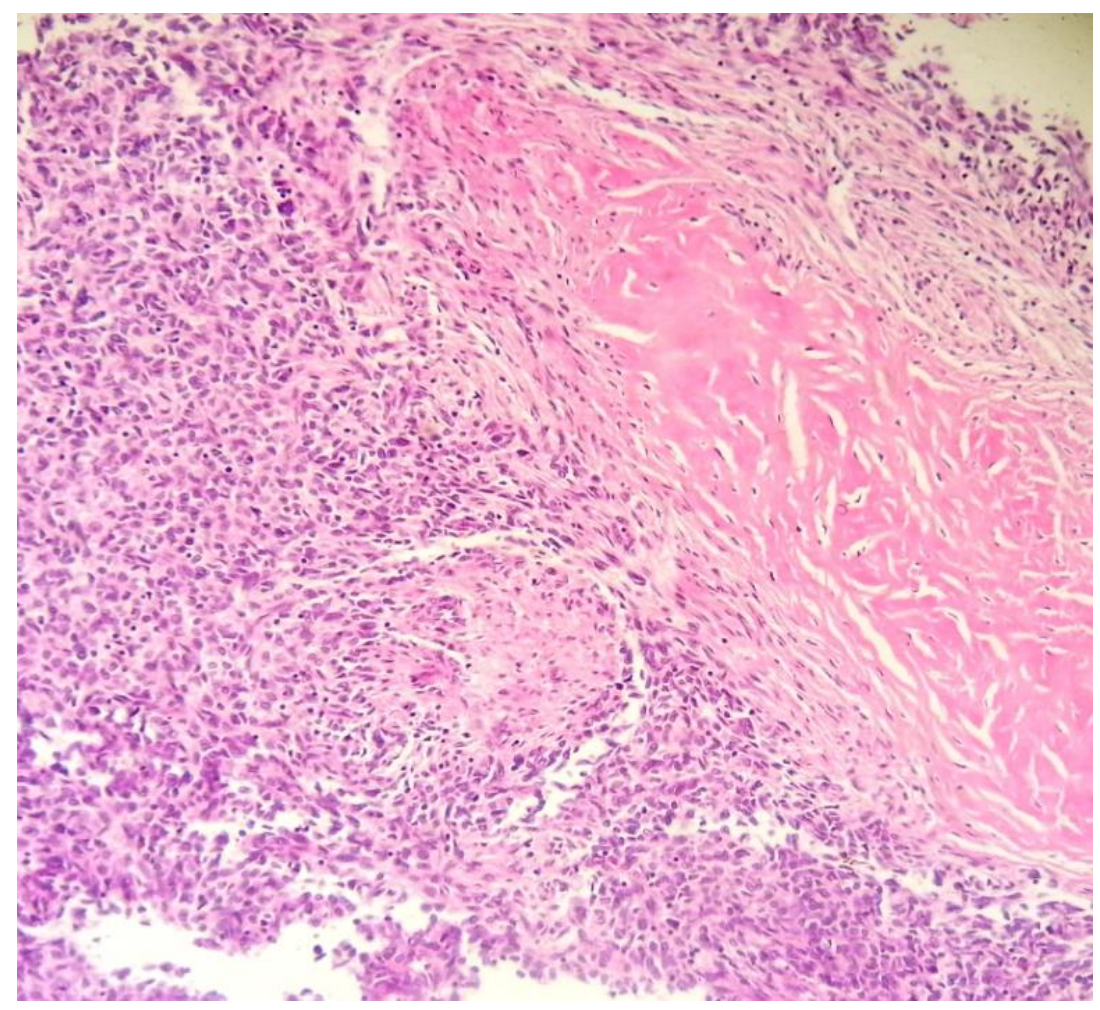

Figure 3: Pathologic evaluation showing fibroconnective tissue and skeletal muscle with diffuse infiltration of round cells with mildly pleomorphic nuclei, scanty to moderate cytoplasm, high nucleus-to-cytoplasm ratio, scattered mitosis, and vascular background 


\section{Discussion}

Glomus tumor arises from the glomus body, a neuromyoarterial unit found within the reticular dermis functioning as a specialized arteriovenous anastomosis (6). The arterial end of the glomus body is encircled by the modified smooth muscle cells (glomus cells) acting to control blood flow to the skin in response to temperature alterations $(7,8)$. Histologically, there are three variants of the glomus tumor based on the predominant component; solid with poor vasculature and scant smooth muscle component, angiomatoid (glomangioma) with a predominant vascular component, and glomangiomyoma with prominent vascular and smooth muscle components (9). Recognition of typical glomus tumor areas is the most important clue for the diagnosis of a symplastic glomus tumor. Typical glomus tumor areas can be usually found on the periphery of the lesion. However, some typical features revealed by the histological examination, such as prominent branching capillary vasculature, perivascular arrangement of the tumor cells, uniform cell shape and size, presence of distinct cell borders, and uniform cellular investment by basement, suggest the glomus tumor (10). In certain difficult cases, the immunohistochemical assay for smooth muscle actin and type IV collagen can be useful for the diagnosis (10). Malignant transformation is very rare. Glomus bodies are most highly clustered in the digits, palms, and soles of the feet and glomus tumors are most commonly described in the upper extremity and especially in the digital subungual area (11). While extradigital glomus tumors are not a rare subgroup of the glomus tumors and various locations have been described (11), facial location is rare and only a few cases have been reported in the literature (11-13). The signs and symptoms of the facial glomus tumor may vary from an asymptomatic (rarely) subcutaneous nodule to excruciating chronic facial pain. The most expected presentation is that of a small, painful, and subcutaneous nodule. A 4:1 male predominance has been reported for the extradigital glomus tumors (13). The widely preferred treatment for the glomus tumor is complete meticulous surgical excision with different available approaches. Histological examination is necessary to confirm the diagnosis. Tumor recurrence varies from 5 to $50 \%$, depending on surgical procedures and the surgeon's skill (14).

We believe that the present glomangioma is one of the largest reported cases. Due to a big size and uncertain borders in some locations during the surgery, recurrence of the tumor can be expected. Due to rarity and lack of reported cases, substantial management of this tumor in the face is unclear; however, surgical treatment is the most helpful therapeutic option. Further reports of this clinical entity are required to achieve a better diagnosis and to improve care of these patients. 


\section{References}

1. McDermott EM, Weiss AP. Glomus tumors. J Hand Surg Am. 2006;31:1397-1400.

2. Campanacci M. Bone and soft tissue tumors: clinical features, imaging, pathology and treatment. New York: Springer-Verlag Wien; 1999.

3. Weidman FD, Wise F. Multiple glomus tumors of the order of telangiectases. Arch Derm Syphilol. 1937;35:414-426.

4. Fornage BD. Glomus tumors in the fingers: diagnosis with US. Radiology. 1988;167:183-185.

5. Kamarashev J, French LE, Dummer R, Kerl K. Symplastic glomus tumor - a rare but distinct benign histological variant with analogy to other 'ancient' benign skin neoplasms. J Cutan Pathol. 2009;36:1099-1102.

6. Fletcher CD. The evolving classification of soft tissue tumours: an update based on the new WHO classification. Histopathology. 2006;48:3-12.

7. Venkatachalam MA, Greally JG. Fine structure of glomus tumor: similarity of glomus cells to smooth muscle. Cancer. 1969;23:1176-1184.

8. Fletcher CD, Unni KK, Mertens F, editors. World Health Organization classification of tumours. Pathology and genetics of tumours of soft tissue and bone. Lyon: IARC Press; 2002.

9. Gombos Z, Zhang PJ. Glomus tumor. Arch Pathol Lab Med. 2008;132:1448-1452.

10. Folpe AL, Fanburg-Smith JC, Miettinen M, Weiss SW. Atypical and malignant glomus tumors: analysis of 52 cases, with a proposal for the reclassification of glomus tumors. Am J Surg Pathol. 2001;25:1-12.

11. Schiefer TK, Parker WL, Anakwenze OA, Amadio PC, Inwards CY, Spinner RJ. Extradigital glomus tumors: a 20-year experience. Mayo Clin Proc. 2006;81:1337-1344.

12. Wang B, Wang J, Shehan J, Sarma DP. Glomus tumor of the cheek. Internet J Dermatol. 2008;6(2).

13. Mounayer C, Wassef M, Enjolras O, Boukobza M, Mulliken JB. Facial "glomangiomas": large facial venous malformations with glomus cells. J Am Acad Dermatol. 2001;45:239-245.

14. Chen SH, Chen YL, Cheng MH, Yeow KM, Chen HC, Wei FC. The use of ultrasonography in preoperative localization of digital glomus tumors. Plast Reconstr Surg. 2003;112:115-119. 